\title{
Plantes à usage médicinale en élevage d'ovins à Sinématiali (Nord de la Côte d'Ivoire)
}

*KONE Kéassemon Hervé Cédessia ${ }^{1}$, COULIBALY Kiyinlma ${ }^{1}$, KONAN Kouakou Severin ${ }^{2}$

${ }^{1}$ Département de Biologie Végétale, UFR des Sciences Biologiques, Université Péléfero Gon Coulibaly BP 1328 Korbogo

¿Département Biocbimie-Génétique, UFR des Sciences Biologiques, Université Pelefero Gon Coulibaly BP 1328 Korbogo

* Auteur correspondant : Cel. : +225 48867530 Email : hervekone2001@gmail.com

Keywords: Ethnovetenary, Medicinal plants, breeders, Sinématiali, Côte d'Ivoire.

Mots-clés : Ethnovétérinaire, Plantes médicinales, Eleveurs, Sinématiali, Côte d'Ivoire.

Publication date 31/07/2019, http://www.m.elewa.org/JAPS

1 RESUME

L'objectif de cette étude est de contribuer à la connaissance et à la préservation des plantes utilisées par les éleveurs pour le traitement des pathologies ovines dans le département de Sinématiali. En effet, l'élevage des ovins et caprins dans la région du Poro est confronté à de nombreux problèmes de santé animale dont la plupart sont des maladies gastriques et parasitaires. Pour résoudre ces problèmes, les éleveurs font recours dans la majorité des cas aux plantes médicinales naturelles. Afin d'identifier ces plantes, des enquêtes ethnobotaniques ont été réalisées à l'aide de fiches de questionnement auprès de 40 éleveurs adultes et expérimentés du département. Les enquêtes effectuées ont permis d'inventorier 24 espèces végétales réparties en 24 genres et 18 familles botaniques. Ces espèces entrent dans la composition de 25 recettes de traitement thérapeutique de divers maux des ovins dont les plus fréquents sont la diarrhée, le mal de ventre, les vers intestinaux et le paludisme. Les espèces entrant dans les diverses compositions appartiennent majoritairement à la famille botanique des Caesalpiniaceae. Les feuilles sont les organes les plus utilisées (52\%). La forme d'utilisation de ces plantes est la décoction (72\%). Ce travail est une étude préliminaire devant servir de base à des études et analyses pharmacologiques plus approfondies des plantes recensées.

\begin{abstract}
The objective of this study is to contribute to the knowledge and the preservation of the plants used by the breeders for the treatment of ovine pathologies in the department of Sinématiali. Sheep and goat farming in the Poro region is faced with many animal health problems, most of which are gastric and parasitic diseases. To solve these problems, breeders resort in most cases to natural medicinal plants. In order to identify these plants, ethnobotanical surveys were conducted using questionnaires from 40 adult and experienced breeders in the department. The surveys carried out made it possible to inventory 24 plant species divided into 24 genera and 18 botanical families. These species form part of recipes for the therapeutic treatment of various ailments of sheep, the most frequent of which are diarrhea, stomach ache, intestinal worms and malaria. The species entering the various compositions belong mainly to the botanical family of Caesalpiniaceae. Leaves are the most used organs $(52 \%)$. The form of use of these plants is the decoction $(72 \%)$. This work is a preliminary study to serve as a basis for further pharmacological studies and analyzes of the identified plants.
\end{abstract}




\section{INTRODUCTION}

Le développement de l'élevage et l'augmentation de la production animale est l'un des enjeux majeurs de la politique nationale d'investissement agricole en Côte d'Ivoire. Cependant, le pays se trouve toujours fortement dépendant de l'extérieur dans ses approvisionnements en viande (Diallo, 2007). Selon le ministère des ressources animales et halieutiques, pour la seule année 1994, le gouvernement ivoirien a dépensé plus de 60 Milliards pour approvisionner sa population en protéines animales. L'élevage en côte d'Ivoire est confronté à de nombreux problèmes de santé animale dont la plupart sont des problèmes gastriques et maladies parasitaires (MIRAH, 2010). En effet, les pertes causées par les nématodes et les parasites gastro-intestinaux sont considérables. Elles sont les principales causes de mortalité et d'importantes pertes économiques dans les systèmes d'élevage bovin et ovin (Keita, 2007). Par ailleurs, le manque d'encadrement sanitaire adéquat, l'insuffisance de fourrage, la transhumance en saison sèche et les conflits récurrents entre éleveurs et agriculteurs concourent également à la réduction de la production (Diallo, 2007). Pour résoudre les problèmes de santé des animaux d'élevage, les populations du Nord en général et ceux du Département de Sinématiali en particulier, font recours à la médecine traditionnelle. En effet, les plantes médicinales constituent le moyen le plus utilisé en milieu rural pour résoudre les problèmes de santé publique. Selon l'OMS (2002), plus de $80 \%$ de la population africaine a recours aux plantes pour ses besoins en soins de santé. En médecine vétérinaire aussi, la phytothérapie est largement sollicitée par les éleveurs

\section{MATERIEL ET METHODES}

3.1 Milieu d'étude : L'enquête a été menée dans la région du Poro précisément dans le Département de Sinématiali. D’une superficie de $13.400 \mathrm{~km}^{2}$, cette région fait partie du Nord de la Côte d'Ivoire. Elle est située entre les parallèles $8^{\circ} 26$ et $10^{\circ} 27$ de latitude Nord et $5^{\circ} 17$ et $6^{\circ} 19$ de longitude Ouest. Elle est limitée au Nord par la traditionnels. La médecine ethno-vétérinaire (MEV) est un terme scientifique pour les soins de santé animale traditionnelle qui englobe les connaissances, les compétences, les méthodes, les pratiques et les croyances concernant les animaux vivants au sein des communautés (McCorkle, 1986). Vu la facilité à préparer et à administrer les médicaments à base de plantes et sa gratuité, la médecine ethno-vétérinaire occupe une place importante dans les pratiques des éleveurs des pays en voie de développement (Merazi et al., 2016). En Côte d'Ivoire, des travaux ont montré l'intérêt de l'usage des plantes en santé animale (Aké Assi et Guinko, 1991 ; Koné et Kamanzi Atindehou, 2008). Ces travaux ont révélé que la flore ivoirienne possède des espèces végétales susceptibles de jouer un rôle dans le contrôle des pathologies bovines et ovines. Par ailleurs, l'absence d'un service vétérinaire dans le Département de Sinématiali, est un véritable problème pour le traitement des animaux d'élevage. Pour pallier à ce problème, les éleveurs de la localité ont recours aux plantes médicinales. Il apparait donc urgent d'identifier ces plantes. L'objectif général du présent travail est de connaitre les plantes utilisées par les éleveurs du Département de Sinématiali pour soigner les pathologies ovines les plus récurrentes. De façon plus spécifique, il s'agira :

(1)-d'identifier les pathologies ovines qui sévissent dans le Département ;

(2)-de recenser les plantes utilisées pour soigner les différentes pathologies ovines ;

(3)-de présenter les organes des plantes couramment utilisés.

(4)-de présenter le mode de préparation et d'administration des remèdes

République du Mali, au Sud par la Région du Béré, à l'Est par les Régions du Tchologo et du Hambol et à l'Ouest par la Région de la Bagoué. Son climat est de type soudanais, marqué par une alternance de deux saisons : saison sèche très marquée par l'harmattan entre décembre et janvier avec une des pointes de chaleur en mars et avril. Saison des pluies 
qui s'étend de mai à octobre avec des pluviométries maximales en juillet et août. La température varie entre 24 et 34 degrés. (Anonyme 1, 2019). Sinématiali se situe à $27 \mathrm{~km}$ de Korhogo et à 620 $\mathrm{km}$ d'Abidjan. C'est une zone propice à l'élevage des bovins et ovins. La végétation du département, comme celle de toute la région, est la savane arborée ou savane ouest soudanienne. Elle se caractérise par des arbres et arbustes, d'une hauteur comprise entre 8 et $12 \mathrm{~m}$, disséminés avec une densité de couvert de l'ordre de 25 à $35 \%$ (Anonyme 2, 2019). La médecine ethno-vétérinaire occupe une place non négligeable dans les pratiques des éleveurs dans la région du Poro et en particulier ceux de Sinématiali. La principale langue parlée en ville et dans les villages environnants est le Nanfara (figure 1).
3.2 Matériel végétal et technique: Le matériel végétal est représenté par l'ensemble des plantes faisant l'objet de cette étude. Le matériel technique est constitué essentiellement d'une fiche d'enquête, d'un gps pour la géolocalisation et d'une moto pour les déplacements.

3.3 Enquêtes ethno-vétérinaires : Au cours de l'enquête, 40 guérisseurs et éleveurs d'ovins matures et expérimentés ont été visités et questionnés. Les informations collectées concernent les pathologies fréquemment rencontrées, les plantes utilisées pour traiter ces pathologies (noms vernaculaires et échantillons, les organes ou parties de la plante utilisée, le mode de préparation et d'administration.

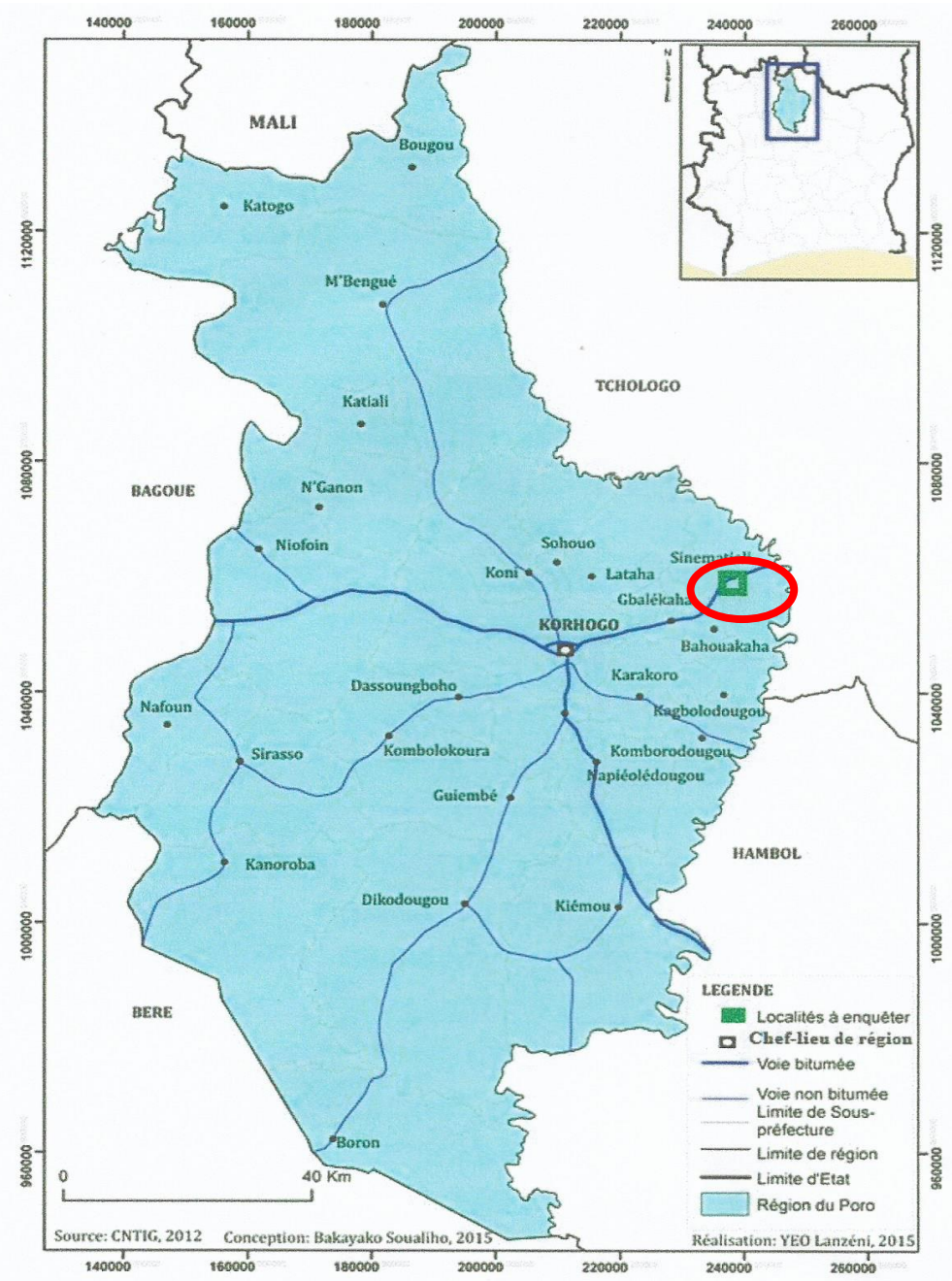

Source : CNTIG, 2012

Figure 1 : Carte de la région du Poro (Côte d'Ivoire) 


\section{$4 \quad$ RESULTATS}

4.1 Pathologies ovines du Département : Les pathologies les plus fréquemment rencontrées en élevage d'ovins dans le Département de Sinématiali sont présentées par la figure 2. Au total 10 pathologies ont été recensées. Ce sont: la diarrhée $(27 \%)$, les vers intestinaux $(12 \%)$ les maux de ventre $(11 \%)$, les zoonoses à plasmodium sp. $(10 \%)$ et infections cutanées par les tiques $(10 \%)$. D'autres pathologies telles que la toux, la constipation, les infections du foie et les fractures ont été observées.

4.2 Plantes médicinales utilisées pour soigner les différentes pathologies ovines : Pour le traitement des pathologies ovines, l'enquête a permis de recenser 19 espèces appartenant à 19 genres répartis entre 15 familles botaniques (Tableau 1). Les familles les plus utilisées sont celles des combretaceae $(16 \%)$ et celle des moraceae avec un taux de 12\% (Figure 3). Les espèces les plus utilisées de la famille des combretaceae sont Anogeissus leiocarpus et Guiera senegalensis. Dans celle des moraceae, Ficus vallis-choudae et Ficus glumosa font l'objet d'un usage courant par les éleveurs et guérisseurs du département. Les parties des plantes utilisées pour le traitement des différentes pathologies sont données par le tableau 2. Pour le traitement des vers intestinaux recours est fait aux espèces Trema guineensis, Cassia siamea et Carica papaya. Pour le traitement des douleurs abdominaux et constipation chez les ovins, les éleveurs et guérisseurs emploient couramment Mitragyna inermis, Diospyros mespiliformis et Entada africana. Les espèces Carica papaya et Anogeissus leiocarpus sont utilisées pour le traitement du paludisme. Le traitement de la toux est aussi une préoccupation pour les éleveurs du département. Ils utilisent comme remèdes des extraits d'écorce de Parkia biglobosa et de Guiera senegalensis. L'espèce Securidaca longipedunculata (Polygalaceae) appelé «sayiri » dans le dialecte local est utilisé pour le traitement des morsures de serpent. Les espèces Sclerocarya birrea et Ficus vallischoudae sont utilisées respectivement pour le traitement des plaies infectées et de la gale.

\title{
Fréquence des pathologies ovines (\%)
}

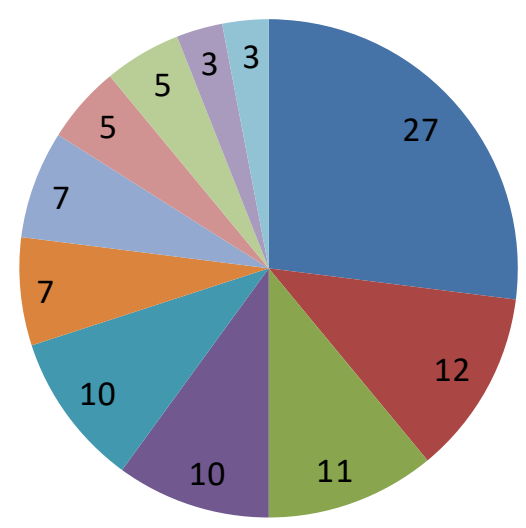

\author{
- Diarhée \\ - vers intestinaux \\ - Douleurs abdominales \\ - zoonoses à plasmodium sp. \\ Tiques \\ - Toux \\ Constipation \\ - Infection du foie \\ - Fièvre \\ Fractures \\ Plaies infectées
}

Figure 2 : Pathologies les plus fréquemment rencontrées en élevage d'ovins 
Tableau 1: Espèces et familles de plantes utilisées par les éleveurs

\begin{tabular}{|c|}
\hline Espèces \\
\hline Adansonia digitata $\mathrm{L}$. \\
\hline Anogeissus leiocarpus (DC.) Guill. \& Perr. \\
\hline Carica papaya L. \\
\hline Cyperus articulatus L. \\
\hline Detarium senegalense J.F.Gmel. \\
\hline Diospyros mespiliformis Hochst. ex A.DC. \\
\hline Entada africana Guill. \& Perr. \\
\hline Ficus glumosa Delile \\
\hline Ficus vallis-choudae Delile \\
\hline Guiera senegalensis J.F.Gmel. \\
\hline Hymenocardia acida Tul. \\
\hline Lannea acida Engl. \& K.Krause \\
\hline Lophira lanceolata Tiegh. ex Keay \\
\hline Mitragyna inermis (Willd.) Kuntze \\
\hline Parkia biglobosa (Jacq.) R.Br. ex G.Don \\
\hline Psidium guajava $\mathrm{L}$. \\
\hline Sclerocarya birrea (A.Rich.) Hochst. \\
\hline Securidaca longipedunculata Fresen. \\
\hline Trichilia emetica Vahl \\
\hline $\begin{array}{l}\text { 4.3 Parties des plantes utilisées : La } \\
\text { fréquence d'utilisation des organes des plantes est } \\
\text { donnée par la figure } 4 \text {. Les remèdes sont } \\
\text { confectionnés le plus souvent à partir des feuilles } \\
(43,33 \%) \text {, des racines }(23,33 \%) \text {, des écorces de } \\
\text { tronc }(23,33 \%) \text {. Les autres parties des plantes } \\
\text { notamment tubercules, graines et tiges sont très peu } \\
\text { utilisées à raison de } 3,33 \% \text { chacune; soit un taux } \\
\text { d'usage total de } 9,99 \% \text {. }\end{array}$ \\
\hline
\end{tabular}

\begin{tabular}{ll}
\hline Familles \\
\hline Bombacaceae \\
\hline Combretaceae \\
Caricaceae \\
\hline Cyperaceae \\
\hline Caesalpiniaceae \\
Ebenaceae \\
\hline Mimosaceae \\
\hline Moraceae \\
\hline Moraceae \\
\hline Combretaceae \\
\hline Phyllanthaceae \\
\hline Anacardiaceae \\
\hline Ochnaceae \\
\hline Rubiaceae \\
\hline Mimosaceae \\
Myrtaceae \\
Anacardiaceae \\
\hline Polygalaceae \\
Meliaceae
\end{tabular}

4.4 Mode de préparation et d'administration: Les organes végétaux frais ou sec, provenant d'une plante ou d'une association de plantes, sont utilisés majoritairement sous forme de décoctions. Les ingrédients tels que le sel, le citron, le gingembre, et le miel entrent dans la confection de certaines recettes. Les remèdes sont administrés par voie orale dans la majorité des cas. Pour les plantes identifiées, les guérisseurs et les éleveurs n'ont mentionné aucun effet secondaire. 


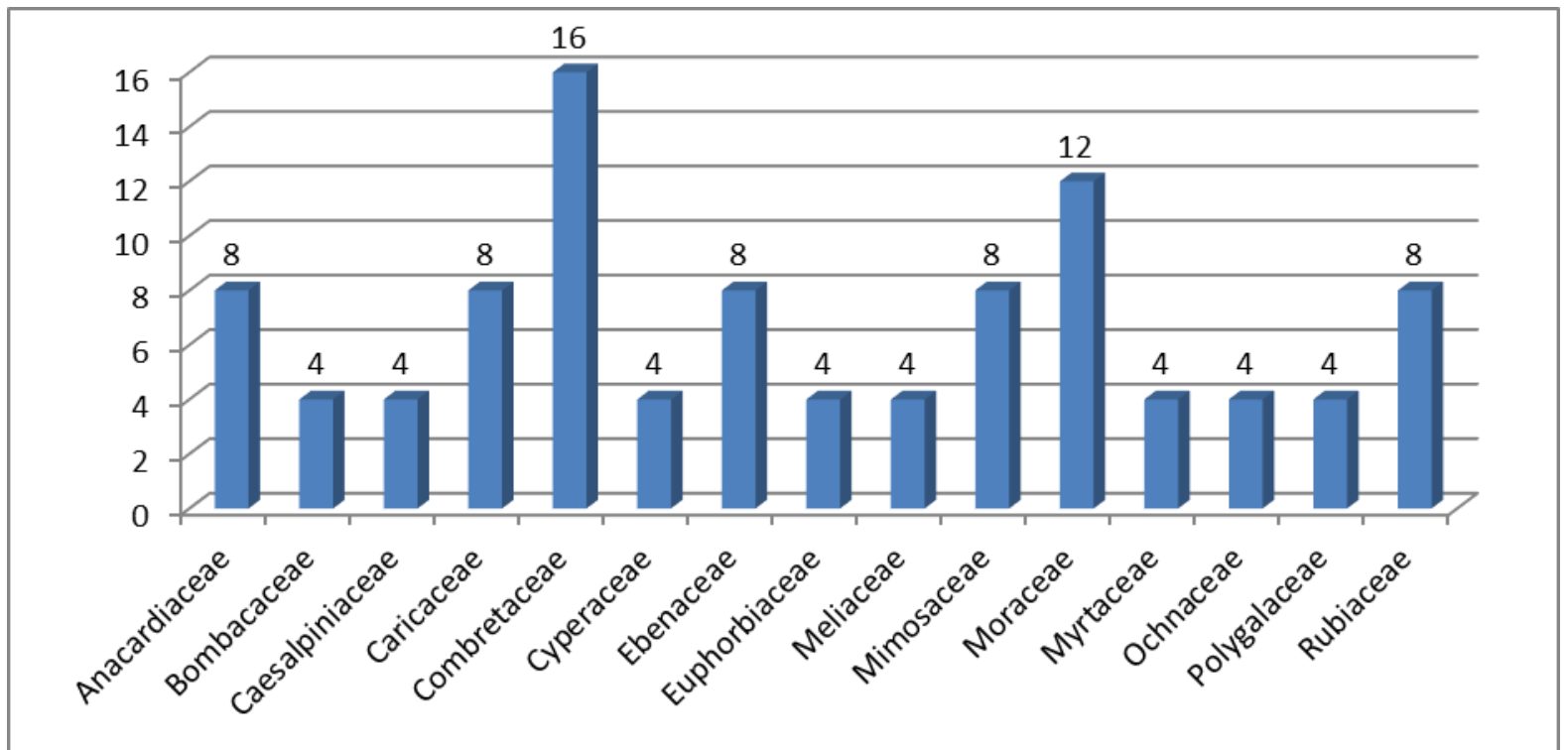

Figure 3 : Fréquence d'utilisation des familles par les éleveurs du Département (\%)

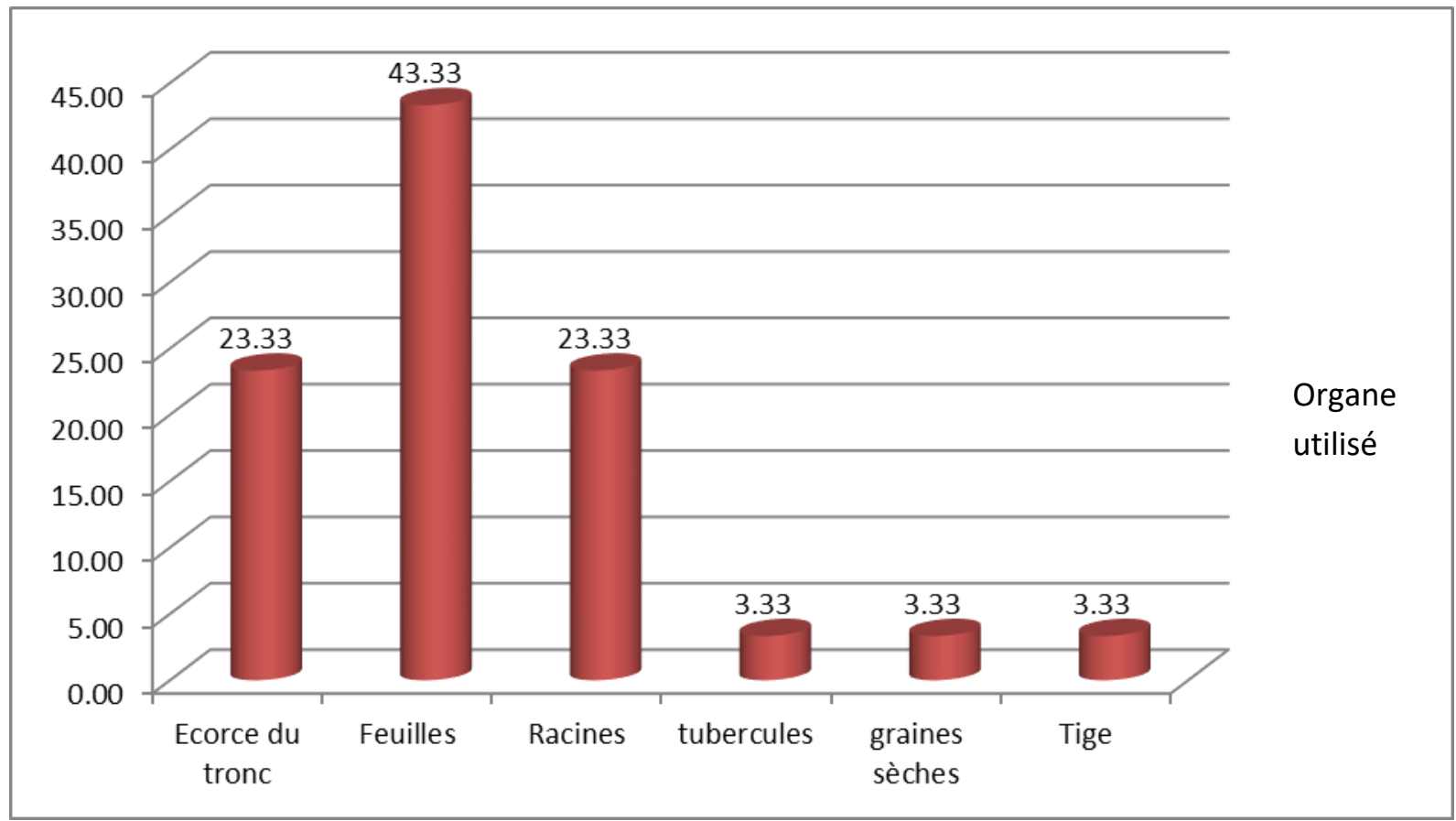

Figure 4 : Fréquence d'utilisation des organes des plantes 
Tableau 2: Mode de préparation et d'administration des remèdes à base de plantes utilisées pour traiter les différentes pathologies ovines dans le Département de Sinématiali.

\begin{tabular}{|c|c|c|c|c|c|c|}
\hline Espèces & Familles & Noms locaux & Parties utilisées & Pathologies & $\begin{array}{c}\text { Mode de } \\
\text { préparation }\end{array}$ & $\begin{array}{c}\text { Mode } \\
\text { d'administration }\end{array}$ \\
\hline Adansonia digitata $\mathrm{L}$. & Bombacaceae & Zihintegué & Ecorce du tronc & Diarrhée & Décoction & Boisson \\
\hline \multirow{2}{*}{$\begin{array}{l}\text { Anogeissus leiocarpus (DC.) } \\
\text { Guill. \& Perr. }\end{array}$} & \multirow[t]{2}{*}{ Combretaceae } & \multirow{2}{*}{$\begin{array}{l}\text { Guenmin/ } \\
\text { N'galama }\end{array}$} & Ecorce du tronc & Toux & Décoction & Boisson \\
\hline & & & Feuilles/tiges/racines & $\begin{array}{l}\text { Infection du } \\
\text { foie }\end{array}$ & Décoction & Boisson \\
\hline \multirow[t]{2}{*}{ Carica papaya L. } & \multirow[t]{2}{*}{ Caricaceae } & \multirow[t]{2}{*}{ Mahandji } & Graines sèches & Vers intestinaux & Décoction & Boisson \\
\hline & & & Feuilles & $\begin{array}{l}\text { Zoonoses à } \\
\text { Plasmodium sp. }\end{array}$ & Macération & Boisson \\
\hline Cyperus articulatus L. & Cyperaceae & Mandja & Tubercules & Diarrhée & $\begin{array}{l}\text { Décoction de la } \\
\text { poudre }\end{array}$ & Boisson \\
\hline $\begin{array}{l}\text { Detarium senegalense } \\
\text { J.F.Gmel. }\end{array}$ & Caesalpiniaceae & Palcthoi & Feuilles & Diarrhée & Décoction & Boisson \\
\hline \multirow{2}{*}{$\begin{array}{l}\text { Diospyros mespiliformis } \\
\text { Hochst. ex A.DC. }\end{array}$} & \multirow[t]{2}{*}{ Ebenaceae } & \multirow[t]{2}{*}{ Gnantchou } & \multirow[t]{2}{*}{ Feuilles } & Constipation & Décoction & Boisson \\
\hline & & & & $\begin{array}{l}\text { Douleurs } \\
\text { abdominales }\end{array}$ & Décoction & Boisson \\
\hline $\begin{array}{l}\text { Entada africana Guill. \& } \\
\text { Perr. }\end{array}$ & Mimosaceae & Yiriwaha & Racines & $\begin{array}{l}\text { Douleurs } \\
\text { abdominales et } \\
\text { constipation }\end{array}$ & Décoction & Boisson \\
\hline Ficus glumosa Delile & Moraceae & Sidjènefielguè & Feuilles & Vers intestinaux & Décoction & Boisson \\
\hline \multirow[t]{2}{*}{ Ficus vallis-choudae Delile } & \multirow[t]{2}{*}{ Moraceae } & \multirow[t]{2}{*}{ Liyirimin/ Yibe } & Ecorce du tronc & Diarrhée & Décoction & Boisson \\
\hline & & & Feuilles & Gale et tiques & Macération & $\begin{array}{l}\text { Epandage sur la } \\
\text { peau }\end{array}$ \\
\hline \multirow[t]{2}{*}{$\begin{array}{l}\text { Guiera senegalensis } \\
\text { J.F.Gmel. }\end{array}$} & \multirow[t]{2}{*}{ Combretaceae } & \multirow[t]{2}{*}{ Koungbè } & $\begin{array}{l}\text { Feuilles/Ecorce du } \\
\text { tronc }\end{array}$ & $\begin{array}{l}\text { Zoonoses à } \\
\text { Plasmodium sp. }\end{array}$ & Décoction & Boisson \\
\hline & & & Racines & Toux & Décoction & Boisson \\
\hline Hymenocardia acida Tul. & Euphorbiaceae & Tipkèri & Racines & Diarrhée & Décoction & Boisson \\
\hline $\begin{array}{l}\text { Lannea acida Engl. \& } \\
\text { K.Krause }\end{array}$ & Anacardiaceae & Dihin & Feuilles/racines & Diarrhée & Décoction & Boisson \\
\hline
\end{tabular}


Journal of Animal \& Plant Sciences (J.Anim.Plant Sci. ISSN 2071-7024) Vol.41 (1): 6828-6839. https://doi.org/10.35759/JAnmPISci.v41-1.9

\begin{tabular}{|c|c|c|c|c|c|c|}
\hline $\begin{array}{l}\text { Lophira lanceolata } \\
\text { Tiegh. ex Keay }\end{array}$ & Ochnaceae & Souhonmon & Racines & Diarrhée & Décoction & Boisson \\
\hline \multirow{2}{*}{$\begin{array}{l}\text { Mitragyna inermis (Willd.) } \\
\text { Kuntze }\end{array}$} & \multirow[t]{2}{*}{ Rubiaceae } & \multirow[t]{2}{*}{ Lirimin } & \multirow[t]{2}{*}{ Feuilles } & Vers intestinaux & Décoction & Boisson \\
\hline & & & & $\begin{array}{l}\text { douleurs } \\
\text { abdominales et } \\
\text { constipation }\end{array}$ & Décoction & Boisson \\
\hline $\begin{array}{l}\text { Parkia biglobosa (Jacq.) } \\
\text { R.Br. ex G.Don }\end{array}$ & Mimosaceae & Nèrè & Ecorce du tronc & Toux & Décoction & Boisson \\
\hline Psidium guajava L. & Myrtaceae & Goyavé & Feuilles & Diarrhée & Macération & Boisson \\
\hline $\begin{array}{l}\text { Sclerocarya birrea (A.Rich.) } \\
\text { Hochst. }\end{array}$ & Anacardiaceae & Kuntango & Feuilles / racines & Plaies infectées & Macération & $\begin{array}{l}\text { Epandage sur la } \\
\text { plaie }\end{array}$ \\
\hline \multirow[t]{2}{*}{$\begin{array}{l}\text { Securidaca longipedunculata } \\
\text { Fresen. }\end{array}$} & \multirow[t]{2}{*}{ Polygalaceae } & \multirow[t]{2}{*}{ Sayiri/Felimin } & Racines & $\begin{array}{l}\text { Morsures de } \\
\text { serpent }\end{array}$ & Decoction & Néttoyer la plaie \\
\hline & & & Racines/feuilles & Maux de ventre & Macération & Boisson \\
\hline Trichilia emetica Vahl & Meliaceae & Gotounonssoukro & Racines/feuilles & Constipation & Decoction & Boisson \\
\hline
\end{tabular}




\section{DISCUSSION}

Cette enquête ethnobotanique réalisée auprès de 40 éleveurs du département de Sinématiali a permis de recenser 10 problèmes de santé des ovins. Les plus fréquemment rencontrés sont la diarrhée $(27 \%)$, les vers intestinaux $(12 \%)$ les maux de ventre $(11 \%)$, les zoonoses à plasmodium sp. (10\%) et les infections cutanées par les tiques $(10 \%)$. D'autres pathologies telles que la toux, la constipation, les infections du foie et les fractures ont été observés chez les ovins de la localité. Les diarrhées font partie des maladies courantes en élevage ovin, entrainant des pertes économiques importantes pour les éleveurs, et cela dans de nombreux pays (Williams et Palmer, 2011). En effet, elles peuvent entraîner des retards de croissance impactant les poids de carcasse (Sweeny et al., 2012). La diarrhée chez les ovins est un syndrome multifactoriel : bien que des agents pathogènes soient diagnostiqués dans la plupart des cas, l'apparition de la diarrhée est favorisée par des facteurs de risque comme les facteurs environnementaux, la sensibilité de l'animal (lié à l'état de son système immunitaire) et son statut nutritionnel, ainsi que les pratiques d'élevage et de conduite du troupeau (Martella et al., 2015). Il existe néanmoins des causes exclusivement alimentaires, principalement chez les agneaux sevrés, où aucun agent pathogène ne peut être mis en cause (Daignault et al., 2009). De nombreux pathogènes peuvent entraîner des diarrhées chez les ovins. Il peut ainsi s'agir de bactéries, de virus ou de parasites (protozoaires et nématodes) (Poncelet, 2008). Selon Anonyme 4 (2019) et Andrés et al. (2006), dans la première semaine de vie des agneaux, plusieurs auteurs s'accordent à dire que E.coli est la bactérie la plus mise en cause dans les cas de diarrhée, associée ou non à des virus ou parasites (Millemann et al., 2003). La forte prévalence des tiques dans la région du poro est rapportée par d'autres études. Selon une enquête menée par Yeo et al. (2017) dans la Région, les tiques seraient responsables de nombreuses maladies notamment l'anaplasmose, la babésiose, la theileriose et l'ehrlichiose. En effet, les tiques, ces parasites minuscules lorsqu'ils se fixent dans la peau des mammifères, se gorgent ensuite du sang de leurs victimes et peuvent être les vecteurs de pathologies parfois mortelles. Selon Kéita (2007), outre ces dégâts "directs", ces parasites transmettent des maladies telles que l'anaplasmose, la cowdriose, la dermatophilose, la theileriose, la babesiose, qui déciment les troupeaux. Selon L'hygiène défectueuse des élevages, la chaleur et l'humidité sont des conditions favorables au développement des ectoparasitoses notamment la gale (Keita, 2007). Les animaux présentent des dépilations avec des croûtes sur la tête, autour du nez et sur les oreilles (gale sarcoptique) ou de grandes surfaces dépilées sur le dos et les flancs (gale psoroptique). L'endoparasitisme dans les élevages d'ovins au Nord de la Côte d'Ivoire est une préoccupation pour les éleveurs de la région. Cela est confirmé par une étude réalisée par Achi et al. en 2003. Selon cette étude, Les moutons et les chèvres élevés dans la région des savanes de la Côte d'Ivoire souffrent d'un polyparasitisme permanent dominé par Trichostrongylus colubriformis et Haemonchus spp. auxquels il faut ajouter Oesophagostomum columbianum. Les infestations parasitaires sont une grande contrainte mal appréhendée par beaucoup d'éleveurs de moutons. Malgré le programme de déparasitage interne (5 interventions par an) préconisé par l'ex-Sodepra (Société de Développement des Productions Animales) pour le Nord de la Côte d'Ivoire, avec des médicaments subventionnés à presque $100 \%$, la moitié des mortalités des agneaux est encore imputable aux parasites gastro-intestinaux (Achi et al., 2003). Par ailleurs, la forte prévalence des helminthes gastrointestinaux dans les élevages subsahariens est rapportée dans une étude réalisée au Burkina Faso (Ouédraogo et al., 1992). Pour lutter contre les pathologies sus mentionnées, Certains éleveurs du Département de Sinématiali ont recours à la médecine traditionnelle. L'enquête a permis d'identifier au total 19 plantes médicinales reparties en 19 genres appartenant à 15 familles différentes utilisées dans le traitement des pathologies ovines. Une étude antérieure montre que les populations de Sinématiali utilisent quasiment les mêmes plantes aussi bien pour le traitement des pathologies bovines qu'ovines (Koné et al., 2019). La différence réside dans le mode d'administration et du dosage des remèdes en tenant compte du poids de l'animal à traiter. Parmi les plantes utilisées, l'usage de Carica papaya (Caricaceae) pour le traitement du paludisme et des vers intestinaux semble la plus répandue (Keita et al., 1999). En effet, le papayer possède de nombreuses vertus médicinales. En phytothérapie humaine, on utilise aussi bien le fruit que les feuilles, les graines, le latex ou les racines. Selon la partie utilisée, le papayer est un purgatif, un anti- 
inflammatoire ou un agent ayant des effets positifs sur la digestion (Anonyme 3, 2018 ; Mpoame et Essomba, 2000). En nous référant aux travaux de Sangaré (2015), les plantes identifiées à Sinématiali sont utilisées comme plantes médicinales ailleurs dans la région du Poro. A Ferkessédougou, pour réduire l'incidence des pathologies bovines et ovines, la population utilise couramment les espèces appartenant à la famille des Meliaceae $(15,15 \%)$ et celle des Caesalpiniaceae (12,12\%). Les maladies fréquemment traitées sont la diarrhée et les vers intestinaux. En outre, Carica papaya (Caricaceae), Ficus vallis-chondae (Moraceae), Anogeissus leiocarpus (Combretaceae) et Detarium microcarpum (Caesalpiniaceae) ont été recensées comme remèdes dans le traitement respectif du paludisme, des tiques, et de la diarrhée dans les systèmes d'élevages bovin et ovin à Korhogo (N'gouan, 2015). Pour quelques espèces végétales très utilisées traditionnellement, un lien a été établi entre les indications thérapeutiques et les propriétés déjà montrées dans des travaux antérieurs. C'est le cas d'Anogeissus leiocarpus (Combretaceae) qui est utilisée pour traiter la diarrhée. L'activité anti diarrhéique de cette plante serait due à la forte teneur en tannin et à la présence de la quercétine (Neuwinger, 1994). A travers toute l'Afrique, pour combattre les vers intestinaux et les désordres gastro-intestinaux, les populations ont recours à Annona senegalensis (Annonaceae). Les études menées sur les propriétés chimiques et pharmacologiques de la plante révèlent que l'acide kaurénoïque est responsable de son efficacité sur les maladies, mais cette espèce présente une très forte toxicité selon Neuwinger (1994). En Côte d'Ivoire comme ailleurs en Afrique, Securidaca longepedunculata (Polygalaceae) est très utilisée pour traiter divers problèmes de santé notamment la pasteurellose bovine et les morsures de serpent (Assogba, 1984 ; Bouquet et Debray, 1974 ; Dro et al., 2013). Cette plante, d'un si grand

\section{CONCLUSION}

Ce travail a permis de recenser puis d'identifier quelques plantes à valeur médicinales pour les systèmes d'élevages ovins dans le département de Sinématiali. Il a montré que la flore de ce département renferme encore un riche potentiel de plantes susceptibles de jouer un rôle dans le traitement de diverses maladies. Les plantes couramment utilisées dans le traitement de diverses infections animales sont Sclerocarya birrea intérêt thérapeutique, est hélas toxique. La sécurinine isolée dans les écorces de tronc et les saponines dans les racines seraient responsables de la toxicité de la plante (Neuwinger, 1994). Toutefois, si les écorces de racines sont utilisées sous forme d'un macéré aqueux à $10 \%$, la toxicité aiguë serait pratiquement nulle. Ainsi, Lejoly et al. (1994) conseillent l'administration de cette plante comme remède que si le dosage est fait sous un contrôle strict. On note que le nombre total d'espèces végétales identifiées est relativement faible sur une surface aussi grande que le département de Sinématiali. Plusieurs raisons peuvent expliquer cette observation. Nous pouvons incriminer en premier lieu certaines pratiques culturales qui consistent au défrichement de nouvelles terres pour la mise en place de cultures exigeantes telles l'igname. A cela s'ajoute la coupe intense de bois de chauffe pour la cuisson du "Tchapalo », boisson très appréciée dans la région du Poro. Les coupes concernent de façon générale les jeunes plantes. La faible richesse peut s'expliquer aussi par les feux de brousses, la saison sèche très rude, le changement climatique et la surexploitation ou la surutilisation. Par ailleurs, les mauvaises techniques de récolte des plantes peuvent aussi être à l'origine de la disparition ou de la raréfaction de certaines d'entre elles telles que Sclerocarya birrea (Anacardiaceae), Ficus vallis-choudae (Moraceae) et Annona senegalensis (Annonaceae). Les plantes sont le plus souvent déterrées pour la récolte des racines utilisées pour la confection des remèdes traditionnelles. Cette raréfaction de certaines espèces dans le nord de la Côte d'Ivoire est rapportée également par les études de Dro (2013). Aussi, insistons-nous sur le fait que si rien n'est fait pour protéger la biodiversité, les plantes à usage médicinales pour l'homme et les animaux disparaitront d'ici quelques décennies dans le département voire dans la région du Poro.

(Anacardiaceae), Carica papaya (Caricaceae), Anogeissus leiocarpus (Combretaceae), Cassia siamea (Caesalpiniaceae), et Parkia biglobosa (Mimosaceae). L'étude a aussi montré la rareté de Sclerocarya birrea (Anacardiaceae) du fait de sa forte utilisation. Cette plante est en voie d'extinction dans le nord du pays. Des campagnes de sensibilisation doivent être organisées afin d'éveiller la conscience des paysans et les encourager à étendre leurs pratiques de 
conservation à un plus grand nombre de plantes utiles ou à les domestiquer. Ceci permettrait une bonne dynamique de préservation des plantes

\section{REFERENCES BIBLIOGRAPHIQUES}

Achi Y.L., Zinsstag J., Yèo N., Dea V. et Dorchies PH. 2003. Épidémiologie des helminthoses des moutons et des chèvres dans la région des savanes du Nord de la Côte d'Ivoire. Revue Méd. Vét., 2003, 154, 3, 179-188.

Andrés S, Jiménez A, Sánchez J, Alonso J.M, Gómez L, López F, Rey J., 2006. Evaluation of some etiological factors predisposing to diarrhoea in lambs in "La Serena" (Southwest Spain). Small Ruminant Research 70, 272-275.

Aké-Assi L et Guinko S. 1991. Plantes utilisées en médecine traditionnelle en Afrique de l'ouest. Edition. Roche, Switzerland, 151 pages.

Anonyme 1 2019. ARDCI : https://www.ardcird.org/index.php/vie-des-regions/poro. consulté le 17/07/2019.

Anonyme 22019.2 Wikipedia: https://fr.wikipedia.org/wiki/Korhogo. Consulté le 17/07/2019.

Anonyme 32018.2 Doctissimo: www.doctissimo.fr/html/sante/phytothéra pie/plante-médicinale/papayer.htm.

Consulté le 28 décembre 2018.

Anonyme 4 2019. Gruenberg W. Diarrhea in neonatal ruminants. MSD Veterinary manual.

https://www.msdvetmanual.com/digestiv e-system/intestinal-diseasesinruminants/diarrhea-in-neonatalruminants). Consulté le 15 juillet 2019.

Assogba M.N. 1984. Quelques enquêtes sur la pharmacopée traditionnelle vétérinaire en république du Benin. 13ieme conférence de la société ouest africaine de pharmacologie les 23-24-25 février 1984 à Cotonou, 22 pages

Bouquet A. et Debray M. 1974. Plantes médicinales de la Côte d'Ivoire. Travaux et documents de l'ORSTOM paris $n^{\circ} 32,232$ pages.

Daignault A, Bourassa R, Moreau J., 2009. La diarrhée chez l'agneau, un sujet à "éviter". Symposium ovin, Centre de référence en agriculture et agroalimentaire du Québec, médicinales et limiterait la menace de disparition qui frappe déjà certaines d'entre elles.

13 (https://www.agrireseau.net/ovins/docum ents/Bourassa_Daignault_Moreau_AR.p df)

Diallo Y. 2007. Les Peuls, les Sénoufo et l'État au nord de la Côte d'Ivoire. Problèmes fonciers et gestion du pastoralisme. ", Bulletin de l'APAD, $10 \mid$ 1995. URL: http://apad.revues.org/1131.

Dro B., Soro D., Koné M.W., Bakayoko A. Kamanzi K. 2013. Evaluation de l'abondance de plantes médicinales utilisées en médecine naturelle dans le nord de la Côte d'Ivoire. Journal of Animal and plant sciences, 2013. Vol 17, Issue 3 : 2631-2646.

Kéita K. 2007. Les tiques parasites des ovins dans les élevages des régions du Centre et du Sud de la Cote d'Ivoire. Thèse de Doctorat. 157 pages.

Keita S.M., Anarson J.T., Baum B.R., Camara F., Traoré A.K. 1999. Etude ethnopharmacologique traditionnelle de quelques plantes médicinales anthelminthiques de la Haute-Guinée (République de Guinée) Rev. Méd. Pharm. Afr., Vol. 13, 49 - 65, (1999).

Koné K.H.C., Coulibaly K., Konan K. S. 2019. Identification de quelques plantes utilisées en médecine ethnovétérinaire à Sinématiali (Nord de la Côte d'Ivoire). Journal of Applied Biosciences 135: 13766 - 13774. ISSN 1997-5902.

Koné M. et Kamanzi A. K. 2006. Evaluation de l'Activité Anthelminthique des Plantes médicinales utilisées en Côte d'Ivoire. Pham. Méd.Trad. Afr. Vol. X/V: pp. 55-72.

Lejoly J., Richel T., Van Essche K. 1994. Plantes Africaines utilisées en médecine traditionnelle. Proceeding of the XIIIth Plenary Meeting AETFAT, Malawi, 1: 197217

McCorkle C. M. 1986. An introduction to ethnoveterinary research and developpement. Journal of ethnobiology Vol 6, No1. 129-149 
Martela V., Decaro N., Buonavoglia C. 2015. Enteric viral infections in lambs or kids. Veterinary Microbiology 706, 7p. (https://kundoc.com/pdf-enteric-viralinfectionsin-lambs-or-kids-.html)

Merazi Y., Hammadi K., Fedoul F. 2016. Approche ethno-vétérinaire des plantes médicinales utilisées dans la région de Sidi Bel AbbesAlgérie. European Scientific Journal. Edition 2016 Vol 12. No 18 ISSN 18577881.

Millemann Y, Adjou K, Maillard R, Chartier C. 2003. Les diarrhées néonatales des agneaux et des chevreaux. Le point vétérinaire $\mathrm{n}^{\circ} 233$, p. 22-29.

MIRAH 2010. Atelier de planification opérationnelle du PSRA. Ministère des Ressources animales et halieutiques. Rapport final. $7 \mathrm{p}$

Mpoame M. et Essomba L. I. 2000. Essai de traitement contre les parasitoses gastrointestinales du poulet avec des décoctions aqueuses de graines de papaye (Carica papaya). RevElevMédVét Pays Trop, 53: 2325.

N'gouan B. 2015. Identification de quelques plantes à usage médicinale pour système d'élevage bovin et ovin à Ferkessédougou (Nord de la Côte d'Ivoire), Mémoire de Licence. UFR des sciences biologiques, Université Péléfero Gon Coulibaly. 40 pages.

Nuewinger H. D. 1996. African Ethnobotany Poisons and Drugs Chemistry Pharmacology- Toxicology. Edition Chapman \& Hall, London.Glasgow. Weinheim.Tokyo.Melbume.Madras, 941 p.

Ouedraogo A., Ouattara L., Kaufmann J., Zinsstag J. et Pfister K. 1992. Epidémiologie des nématodes gastro-intestinaux des ruminants au Burkina Faso : Spectre, fréquences et variations saisonnières. In : Septième conférence internationale des institutions de médecine vétérinaire tropicale, Yamoussoukro, Côte d'Ivoire, 14-18 septembre 1992.

OMS. 2002. Stratégie de l'OMS (Organisation Mondiale de la Santé), pour la médecine traditionnelle (20022005).WHO/EDM/TKM/2002. Genève.
Poncelet J.L. 2008. Les diarrhées de l'agneau. sngtv fiche $\mathrm{n}^{\circ} 23$. (http://ovine.sngtv.pagespersoorange.fr/Diarrhees $\% 20$ agneau.pdf)

Sweeny J.P.A, Ryan U.M, Robertson I.D, Jacobson C. 2012. Prevalence and on-farm risk factors for diarrhoea in meat lamb flocks in Western Australia. The Veterinary Journal 192, 503-510

Williams A.R., Palmer D.G. 2011. Interactions between gastrointestinal nematode parasites and diarrhea in sheep : pathogenesis and control. The Veterinary journal 192, 279285.

Yéo N., Karamoko Y., Soro D., Zouh Bi Z. F. et Traoré S. I. 2017. Elevages de bétail dans la région du Poro (Côte d'Ivoire) : Caractérisation et modalités de lutte contre les pathogènes transmis par les tiques. Int. J. Biol. Chem. Sci. 11(1): 237-246, February 2017 ISSN 1997-342X (Online), ISSN 1991-8631 (Print). 